\title{
PROSPECTIVE INTERVENTIONAL STUDY ON CLINICAL OUTCOMES OF LICHTENSTEIN'S TENSION FREE INGUINAL HERNIOPLASTY UNDER LOCAL ANAESTHESIA IN A TERTIARY CARE HOSPITAL IN EASTERN INDIA
}

\author{
Partha Protim Mondal' ${ }^{1}$ Amit Ray², Purba Haldar ${ }^{3}$
}

${ }^{1}$ RMO-Cum-Clinical Tutor, Department of Urosurgery, CMC\&H, Kolkata, West Bengal, India.

${ }^{2}$ Associate Professor, Department of General Surgery, NBMC\&H, Darjeeling, West Bengal, India.

${ }^{3}$ Associate Professor, Department of Anaesthesiology, RGMC\&H, Birbhum, West Bengal, India.

ABSTRACT
BACKGROUND
Repair of inguinal hernia is one of the most common operations performed by general surgeons worldwide. This study
efficacy and acceptability.

\section{METHODS}

A prospective study was conducted in the Department of General Surgery in a tertiary care medical college and hospital. Patients admitted in wards with inguinal hernia from General Surgery Out Patient Department were selected for this study. The series consisted of 60 patients between 15 and 74 years. The study period is from April 2007 to September 2008. These 60 patients had complaints of groin swelling, in right side 35 patients, or left side 23 patients, and both sides 2 patients. Most of them were reducible. All adult patients suffering from inguinal hernia were included in the study. The exclusion criteria were- obstructed or strangulated hernia, extremes of age, terminal malignancy, advanced diabetes, renal failure and psychiatric disorder. Hernioplasty was done under local anaesthesia with an average of $45 \mathrm{ml}$ of mixture of $1 \%$ lidocaine and $0.5 \%$ bupivacaine with or without $1 / 200000$ epinephrine for a unilateral inguinal hernia repair. All patients underwent elective surgery and were analysed with regard to surgical outcome, complications, cost effectiveness, acceptability and hospital stay.

\section{RESULTS}

In our study, 21 cases had early minor complications. There was no recurrence and no mortality.

\section{CONCLUSIONS}

It is an advantageous procedure because of feasibility, efficacy, greater safety, better post-operative pain control, satisfactory patient acceptance, lower operative time, early return to work and reduced cost.

\section{KEY WORDS}

Repair, Inguinal Hernia, Local Anaesthesia, Patients, Cost

HOW TO CITE THIS ARTICLE: Mondal PP, Ray A, Haldar P. Prospective interventional study on clinical outcomes of lichtenstein's tension free inguinal hernioplasty under local anesthesia in a tertiary care hospital in Eastern India. J. Evolution Med. Dent. Sci. 2019;8(24):1904-1908, DOI: 10.14260/jemds/2019/419

\section{BACKGROUND}

Weakening of the abdominal wall tissue as one of the causes of inguinal hernias was suspected by Cooper as far back as 1800. The matter was emphasized again in 1922 by Harrison when he wondered "Why dozens and hundreds of men show hernias at 50 to 60 years after their active life is over". Need for prosthetic reinforcement of weakened abdominal wall tissue was recognized by Billroth, who mused "If only the proper material could be created to artificially produce tissue of density and toughness of fascia and tendon, the secret of the radical cure for hernia could be discovered'.

'Financial or Other Competing Interest': None.

Submission 27-04-2019, Peer Review 01-06-2019,

Acceptance 08-06-2019, Published 17-06-2019.

Corresponding Author:

Dr. Amit Ray,

Flat-L3, Block IV

Swabhumi Residency, P-12,

Motijheel Avenue,

Kolkata-700074,

West Bengal, India.

E-mail: amitraydr@gmail.com

DOI: $10.14260 /$ jemds/2019/419

(c) $($ ) $९$
With the necessity of prosthesis for the repair of inguinal hernia in mind, and focusing on the principle of "no tension" (Considered one of the great principles of surgery by Halsted), the Lichtenstein group popularized routine use of mesh in 1984 and coined the term "Tension free hernioplasty". In the tension free hernioplasty, instead of suturing anatomic structures that are not in apposition, the entire inguinal floor is reinforced by insertion of a sheet of mesh. The procedure addresses both issue of hazardous suture line tension and metabolic causation of inguinal hernias. The operation is therefore therapeutic as well as prophylactic; it repairs and protect the entire susceptible region of the groin to herniation from all future metabolic and mechanical adverse effects. Repair of inguinal hernia is one of the most commonly performed operations world-wide. ${ }^{1}$ It is more common in males than females. Local anaesthesia for groin hernia repair had been introduced at the very beginning of the last century, and gained popularity following the success reports from the Shouldice Hospital, and the Lichtenstein Hernia Institute. ${ }^{2}$ In India it is not widely accepted despite result of several studies indicate that local anaesthesia provides best clinical and economic benefits to patients.3,4 Inguinal hernia repairs performed under local anaesthesia and sedation is a method of less impact on the 
organ function and systems, and is safe, simple, effective, economical, easy to perform, with lower incidence of side effects, such as cardiovascular instability, nausea, vomiting, urinary retention, spinal headache and early return to work and resulting in a shorter hospital stay. ${ }^{2-5}$ Furthermore, local anaesthesia administered before making the incision produces a prolonged analgesic effect via inhibition of the build-up of local nociceptive molecules. Several safe and effective anaesthetic agents are currently available. An average of $45 \mathrm{ml}$ of 50:50 mixture of 1\% Lidocaine and 0.5\% Bupivacaine, with 1:200, 000 epinephrine is usually sufficient for an unilateral hernia repair. At the line of incision, $5 \mathrm{ml}$ drug is infiltrated subdermally to block subdermal nerve endings and to reduce the discomfort of intradermal infiltration and $3 \mathrm{ml}$ drug infiltrated intradermally. With deep subcutaneous injection, $10 \mathrm{ml}$ of mixture is injected into the subcutaneous adipose tissue with vertical insertion of the needle $2 \mathrm{~cm}$ apart. $10 \mathrm{ml}$ of mixture is injected immediately underneath the aponeurosis of external oblique muscle. This injection floods the enclosed inguinal canal and anaesthetizes all three major nerves in this region. Analgesic effect of local anaesthesia can be further prolonged by splashing $10 \mathrm{ml}$ of the mixture into the inguinal canal before closure of the external oblique aponeurosis and I the subcutaneous space before skin closure. The aim of this study to justify feasibility of inguinal hernia repair under local anaesthesia with sedation and analgesia.

\section{METHODS}

A prospective interventional study was conducted in the Department of General surgery in a tertiary medical care institution. Patients admitted in wards with inguinal hernia from General Surgery Out Patient Department were selected for this study. The series consisted of general anaesthesia fit 60 patients between 15 to 74 years who underwent Lichtenstein tension free inguinal hernioplasty under local anaesthesia and intravenous sedation and analgesia. The study period is from April 2007 to September 2008. These 60 patients had complaints of groin swelling in right or left side groin or both sides and reducible.

Considering the formula for sample size, $\mathrm{n}$ (Sample size $)=$ $\mathrm{z} \alpha 2 \mathrm{p}(1-\mathrm{p}) / \mathrm{e} 2$ where $\mathrm{p}$ is proportion, e is precision. here $\alpha=$ $5 \%$ hence $z \alpha=1.96 ; p$ (prevalence of Hernia) $=1.7 \% \mathrm{e}=3.5 \%$. Using these values in the above formula, sample size (n) is coming as 53. However, to account for probable drop outs and based on available data we have included 60 patients will be in the study.

Patients were operated by either visiting surgeons or resident surgeons under supervision of visiting surgeons. Anaesthesia technique was explained to patient before giving the block, particularly about the early infiltration pain and mild dragging sensations during the operation. Prophylactic antibiotic coverage was given by intravenous injection of onegram ceftriaxone before incision and twelve hours after operation. Intravenous sedation and analgesia with intravenous bolus of 2-3 mg of injection midazolam, $3 \mathrm{mg} / \mathrm{kg}$ of Tramadol injection continuous intravenous infusion.

Hernioplasty done under local anaesthesia with an average of $45 \mathrm{ml}$ of mixture of $1 \%$ lidocaine and $0.5 \%$ bupivacaine with or without $1 / 200000$ epinephrine for a unilateral inguinal hernia repair. Lidocaine provides rapid onset of action and bupivacaine results longer duration action. Use of both of these drugs decreases doses of both drugs and toxicity.

The $12^{\text {th }}$ thoracic, iliohypogastric and ilioinguinal nerves were blocked by injection of 10 to $15 \mathrm{ml}$ of anaesthetic solution deep to external oblique aponeurosis $1.5 \mathrm{~cm}$ medial and superior to the anterior superior iliac spine. Genital branch of genitofemoral nerve was blocked by injecting 10 to $15 \mathrm{ml}$ of anaesthetic solution above the mid inguinal point deep to external oblique. Skin and subcutaneous tissue in and around the proposed line of incision was infiltrated with 20 to $30 \mathrm{ml}$ of anaesthetic solution. Local infiltration of 5 to 10 $\mathrm{ml}$ in the midline above pubic tubercle and medial part of external ring. During operation instillation of 5 to $7 \mathrm{ml}$ of solution into the opened hernia sac to be absorbed directly by the mesothelium which helps manipulation of peritoneum. Small amount also instilled into the cord and it facilitates the manipulation of cord. Rest of the prepared solution was kept ready for infiltration deep in operation field when patients experienced pain during operation. During operations patients were closely observed and monitored by experienced anaesthesiologist.

In Lichtenstein, s tension free inguinal hernioplasty polypropylene mesh was used. With the use of this mesh, it is now possible to eliminate formal reconstruction of the canal floor which inevitably causes anatomic distortion of the canal. It also eliminates suture line tension completely. The study is in accordance with the Declaration of Helsinki for research in humans. Informed consent was obtained from patients after thorough discussion about risks and benefits. Permission from Institutional ethics committee (IEC) was obtained before this study. After compilation of all collected data, analysis was done using.

\section{Statistical Analysis}

After compilation of all collected data, analysis is done using Statistical Package for Social Sciences (SPSS), version 20 (IBM, Chicago, USA, ) and. Categorical variables are expressed as Number of patients and percentage of patients and compared using Pearson's Chi Square test for Independence of Attributes/Fisher's Exact Test as appropriate. Continuous variables are expressed as Mean \pm Standard Deviation and compared using unpaired $t$ test/One Way ANOVA. Association between continuous variables are captured by Pearson's Correlation Coefficient. The statistical software SPSS version 20 are used for the analysis.

An alpha level of $5 \%$ has been taken, i.e. if any $p$ value is less than 0.05 it is considered as significant.

\section{RESULTS}

Patients were admitted on the morning of the day of operation. Total data of 60 patients was analysed in this study. Majority of our patients were in the age group of 35-44 years (25\%). Mean age of presentation in our study is 40.78 years and the youngest member was 18 years old and oldest member presented with at the age of 73 years (Figure 1). 34 $(56.67 \%)$ patient presented with inguinal swelling and 26 (43.33\%) patient with inguinoscrotal swelling. 35 (58.33\%) patients had right sided inguinal hernia, 23(38.33\%) patients had left sided hernia and 2 (3.33\%) patients had bilateral hernias. Per operative Nyhas type of hernia, 10 (16.67\%) cases type I, 28 (46.67\%) type II, $13(21.67 \%)$ type III-A, 7 (11.67\%) type III-B, 2 (3.33\%) type IV (Figure 2). All operations were completed successfully. 


\begin{tabular}{|c|c|c|}
\hline Age Group & No. of Cases & Percentage \\
\hline $15-24$ yrs. & 10 & $16.67 \%$ \\
\hline $25-34$ yrs. & 12 & $20 \%$ \\
\hline 35-44 yrs. & 15 & $25 \%$ \\
\hline 45-54 yrs. & 11 & $18.33 \%$ \\
\hline 55-64 yrs. & 7 & $11.67 \%$ \\
\hline 65-74 yrs. & 5 & $8.33 \%$ \\
\hline Total & 60 & $100 \%$ \\
\hline \multicolumn{3}{|c|}{ Table 1. Distribution Age Among Study Population } \\
\hline
\end{tabular}

\begin{tabular}{|c|c|c|}
\hline Symptoms & No. of Cases & Percentage \\
\hline Inguinal & 34 & $56.67 \%$ \\
\hline Inguinoscrotal & 26 & $43.33 \%$ \\
\hline Total & $\mathbf{6 0}$ & $\mathbf{1 0 0 \%}$ \\
\hline Table 2. Type of Inguinal Hernia Among Study Population \\
\hline
\end{tabular}

\begin{tabular}{|c|c|c|}
\hline Side & No. of Patients & Percentage \\
\hline Right & 35 & $58.33 \%$ \\
\hline Left & 23 & $38.33 \%$ \\
\hline Bilateral & 2 & $3.33 \%$ \\
\hline Total & $\mathbf{6 0}$ & $\mathbf{1 0 0} \%$ \\
\hline \multicolumn{2}{|c|}{ Table 3. Side of Inguinal Hernia Among Study Population } \\
\hline
\end{tabular}

\begin{tabular}{|c|c|c|}
\hline Types & Number & Percentage \\
\hline Indirect & 46 & $76.67 \%$ \\
\hline Direct & 11 & $18.33 \%$ \\
\hline Combined & 2 & $1.67 \%$ \\
\hline Recurrent & 2 & $3.33 \%$ \\
\hline Total & $\mathbf{6 0}$ & $\mathbf{1 0 0} \%$ \\
\hline \multicolumn{2}{|c|}{ Table 4. Types of Hernia on Clinical Examination } \\
\hline
\end{tabular}

\begin{tabular}{|c|c|c|}
\hline Type & No. of Cases & Percentage \\
\hline Type I & 10 & $16.67 \%$ \\
\hline Type II & 28 & $46.67 \%$ \\
\hline Type III & 20 & $33.33 \%$ \\
\hline Type IV & 2 & $3.33 \%$ \\
\hline Total & $\mathbf{6 0}$ & $\mathbf{1 0 0} \%$ \\
\hline \multicolumn{2}{|c|}{ Table 5. Preoperative Nyhus Type of Hernia } \\
\hline
\end{tabular}

\begin{tabular}{|c|c|c|}
\hline Gap (cm) & No. of Cases & Percentage \\
\hline$<0.5$ & 5 & 8.33 \\
\hline $0.5-2$ & 41 & 68.33 \\
\hline$>2$ & 16 & 23.33 \\
\hline Total & $\mathbf{6 0}$ & $\mathbf{1 0 0}$ \\
\hline \multicolumn{2}{|c|}{ Table 6. Gap Between Inguinal Ligament and Transverse Arch } \\
\hline
\end{tabular}

\begin{tabular}{|c|c|c|}
\hline Size & No. of Cases & Percentage \\
\hline Normal & 17 & $28.33 \%$ \\
\hline Marginal & 33 & $55 \%$ \\
\hline Wide & 10 & $16.67 \%$ \\
\hline Total & $\mathbf{6 0}$ & $\mathbf{1 0 0 \%}$ \\
\hline Table 7. Preoperative Assessment of Size of Deep Inguinal Ring \\
\hline
\end{tabular}

\begin{tabular}{|c|c|c|}
\hline Type of Pain & No. of Cases & Percentage \\
\hline No pain & 45 & 75 \\
\hline Mild & 12 & 20 \\
\hline Moderate & 3 & 5 \\
\hline Severe & 0 & 0 \\
\hline Total & $\mathbf{6 0}$ & $\mathbf{1 0 0}$ \\
\hline \multicolumn{2}{|c}{ Table 8. Pain Score at 1 Week } \\
\hline
\end{tabular}

\begin{tabular}{|c|c|c|c|}
\hline Age Group & No. of Patients & Well Accepted & Poorly Accepted \\
\hline $15-34$ yrs. & 22 & 12 & 10 \\
\hline $35-54$ yrs. & 26 & 20 & 6 \\
\hline $55-74$ yrs. & 12 & 11 & 1 \\
\hline Total & $\mathbf{6 0}$ & $\mathbf{4 3}(\mathbf{7 1 . 6 7 \% )}$ & $\mathbf{1 7}(\mathbf{2 8 . 3 3 \% )}$ \\
\hline \multicolumn{4}{|c|}{ Table 9. Acceptance of the Method by The Patients } \\
\hline
\end{tabular}
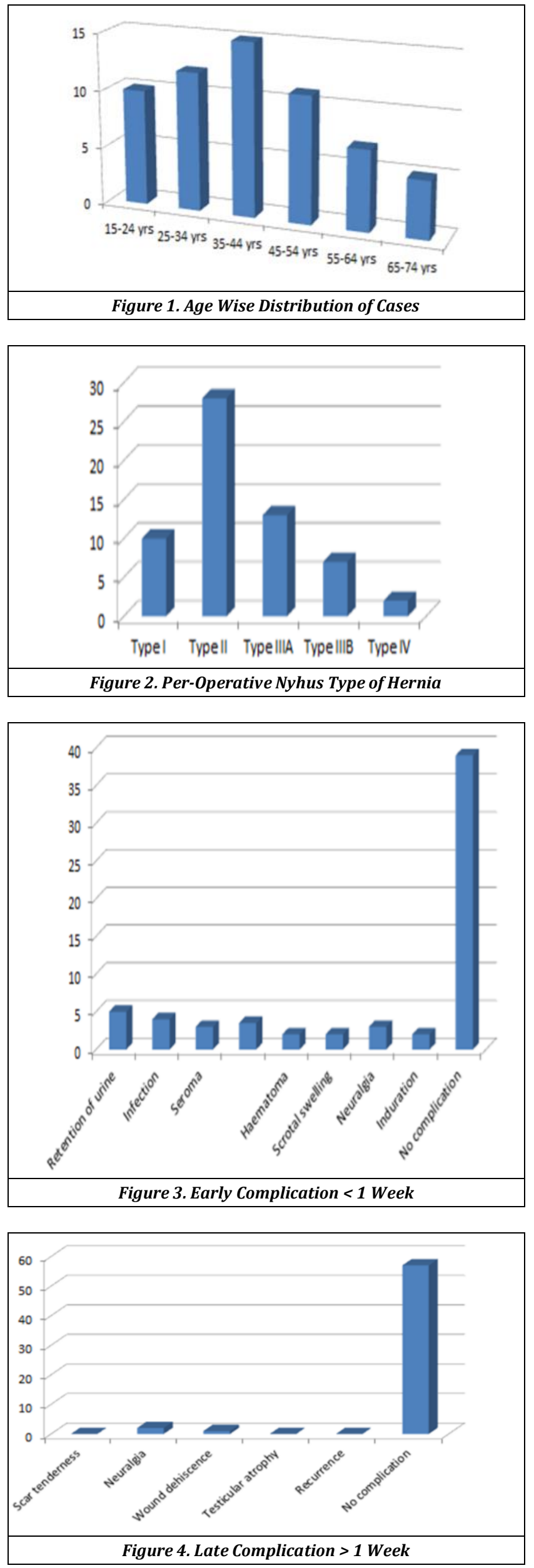

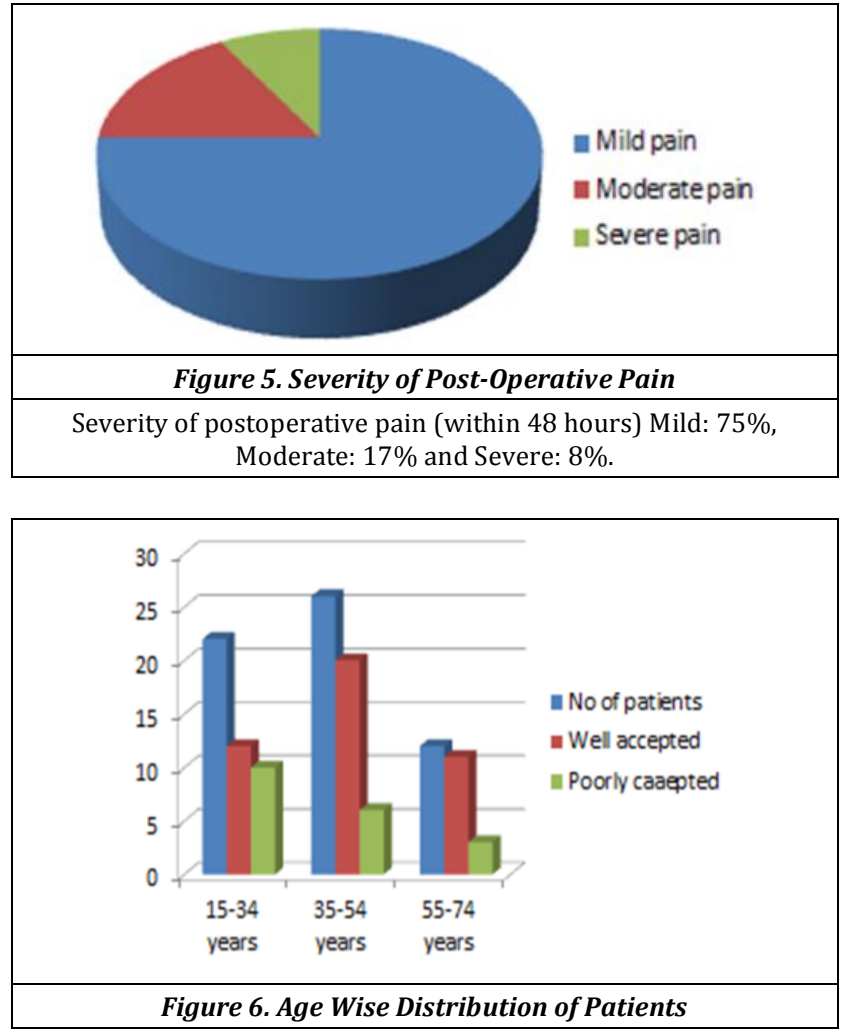

In neither case change of mode of anaesthesia was required. Duration of operation ranged from 45 to 85 minutes, with an average of 65 minutes. In our study 21 (35\%) cases had early complications within one week of which, 2 cases $(3.33 \%)$ had retention of urine, 4 cases (6.67\%) had wound infection, 3 cases $(5 \%)$ had seroma, 2 cases(3.33\%) had haematoma, 2 cases (3.33\%) had scrotal swelling, 3 cases (5\%) had neuralgia and 5 cases(8.33\%) had induration (Figure 3). In our study $3(5 \%)$ cases had late complications after one week of which 2 (3.33\%) had neuralgia and one (1.67\%) had wound dehiscence (Figure 4).

Most of the patient suffering from mild degree of pain 45 cases $(75 \%)$ and 10 cases $(16.67 \%)$ moderate degree of pain and only 5 cases $(8.33 \%)$ suffering from severe degree of pain (Figure 5). Mild to moderate degree of pain is responded by use of oral or parenteral paracetamol or NSAIDS. Severe degree pain requires injectable forms of opioids or tramadol. It was found that it is well accepted in older age group (55-74 years) $(91.67 \%)$ and poorly accepted $(45.45 \%)$ in younger age group (15-34 years) (Figure 6) because younger patient is very much apprehensive about local anaesthesia and postoperative pain. No recurrence has yet occurred in $11 / 2$ years follow up. This $1 \frac{1}{2}$ years period is very much insufficient to judge about the efficacy of any method and also very short to comment about the recurrence. No mortality occurs in this study. Anaesthetic drug related toxicities like hypotension, spinal headache, post-operative nausea and vomiting etc were reported in this study group. 38 (63.33\%) patients were discharged on first post-operative day with oral antibiotics and analgesics. $14(23.33 \%)$ patients on second postoperative and $8(13.33 \%)$ patients on third post-operative days.

\section{DISCUSSION}

Inguinal hernia repair is one of the most common surgical procedure performed in the world. Local anaesthetic repair of inguinal hernia is exercised routinely in a very few specialist centre who are specially interested like Should ice clinic in Toronto, where most of hernias are repaired under local anaesthesia. ${ }^{6}$ A renewed interest in recommending local anaesthesia for inguinal hernia repair, 5,6 has gain popularity after introduction of tension free hernioplasty which is easier to perform than conventional methods of hernia repair. Operative stress had reduced by admitting the patient on the morning of operation as OPD case. ${ }^{7}$ Local anaesthesia along with adjuvant intravenous sedation and analgesia minimises intraoperative and postoperative pain in inguinal hernia surgery. ${ }^{8}$ Urinary retention is a common complication occurs after any anorectal and perineal surgery. ${ }^{5,8}$ Incidence of urinary retention is lower with local anaesthetic inguinal hernia repair than general or regional anaesthesia. ${ }^{9}$ Inguinal hernia repair under local anaesthesia is advantageous in early ambulation, short recovery time no need of endotracheal intubation. ${ }^{8}$ Scrotal haematoma is a common complication in the repair of large hernias. ${ }^{5}$ In our study it was 2 cases $(3.33 \%)$. Inguinal hernia repair is a clean operation with occurrence of wound infection should be is less than one percent. ${ }^{10}$ In our study it was 4 cases $(6.67 \%)$. Post hernia repair chronic groin pain, defined as pain that persists after the normal healing process has occurred typically 3 months after surgery. It is most often a result of nerve injury sustained during improper dissection. Incidence of chronic groin pain following a local anaesthetic inguinal hernia repair is $1 \%$ at 1 -year follow up. ${ }^{11}$ Lidocaine toxicity is usually due to high blood concentration secondary to inadvertent intravenous injection, excessive doses and rapid absorption. ${ }^{12}$ Benefits of local anaesthetic inguinal hernia repair include greater safety profile for the patient, better postoperative pain control, early recovery time, reduced cost compared to hernia repair under general anaesthesia.5,6,7

\section{CONCLUSIONS}

Lichtenstein's tension free inguinal hernioplasty under local anaesthesia is safe and without any untoward effects like post-operative nausea and vomiting, urinary retention, spinal headache and cardio-vascular instability. Inguinal hernia repair under local anaesthesia allows the patient's immediate mobilization, early return to work, minimum discomfort of patients, low or no recurrence and hence cost effective. Short learning curve and simplicity of operation irrespective of type of hernias like direct, indirect, combined and recurrent makes it less operator's experience dependent. Inguinal hernia repair and in those operations where pain due to visceral origin is minimum and where profound muscle relaxation is not required, local anaesthesia is beneficial and has become the anaesthesia of choice among patients.

\section{REFERENCES}

[1] Baskerville PA, Jarret PE. Day case inguinal hernia repair under local anaesthetic. Ann R Coll Surg Engl 1983;65(4):224-5.

[2] Kulacoglu H, Alptekin A. Current options in local anaesthesia for groin hernia repairs. Acta Chir IugosI 2011;58(3):25-35.

[3] Gonullu NN, Cubukcu A, Alponat A. Comparison of local and general anesthesia in tension-free (Lichtenstein) hernioplasty: a prospective randomized trial. Hernia 2002;6(1):29-32. 
[4] Nordin P, Zetterstrom H, Gunnarsson U, et al. Local, regional or general anaesthesia in groin hernia repair: multicentre randomized trial. The Lancet 2003;362(9387):853-8.

[5] Amid PK, Shulman AG, Lichtenstein IL. Local anesthesia for inguinal hernia repair step-by-step procedure. Annals of Surgery 1994;220(6):735-7.

[6] Peiper C, Tons C, Schippers E, et al. Local versus general anaesthesia for Shouldice repair of the inguinal hernia. World J Surg 1994;18(6):912-6.

[7] Glasgow F. Inguinal hernia repair using local anaesthesia. Ann Roy Coll Surg Eng 1984;66(6):382-7.

[8] Teasdale C, McCrum AM, Williams NB, et al. Randomised controlled trial to compare local with general anaesthesia for short-stay inguinal hernia repair. Ann Roy Coll Surg Eng 1982;64(4):238-42.
[9] Kark AE, Kurzer MN, Belsham PA. Three thousand one hundred seventy-five primary inguinal hernia repairs; advantages of ambulatory open mesh repair using local anaesthesia. Journal of the American College of Surgeons 1998;186(4):447-56.

[10] Young DV. Comparison of local spinal and general anaesthesia for inguinal herniorrhaphy. Am J Surg 1987;153(6);560-3.

[11] Sanjay P, Woodward A. Inguinal hernia repair: local or general anaesthesia? The Annals of the Royal College of Surgeons of England 2007;89(5):497-503.

[12] Butterworth JF 4th, Strichartz GR. Molecular mechanism of local anaesthesia: a review. Anaesthesiology 1990;72(4):711-34. 